\title{
High-precision automated testing system for wireless sensor network IC Xingcheng Zhang ${ }^{1, a^{*}}$, Xuan Tang ${ }^{1, b}$, Zhen Meng ${ }^{1, c}$, Mou Liu ${ }^{1, d}$, Yuepeng Yan ${ }^{1, e}$ \\ 1 Institute of Microelectronics of Chinese Academy of Sciences, Beijing, 100029, P. R. China \\ azhangxingcheng@ime.ac.cn, btangxuan@ime.ac.cn, ${ }^{\mathrm{c}}$ mengzhen@ime.ac.cn, \\ dliumou@ime.ac.cn, èyanyuepeng@ime.ac.cn \\ * Corresponding author: zhangxingcheng@ime.ac.cn
}

Keywords: Internet of Things, Wireless Sensor Network, Automated Test, High Precision, RF, IC Test

\begin{abstract}
This paper introduces a high precision automated test system. We built an automated testing platform using programmable test equipments and designed a series of test boards to adapt the test process. Combined virtual instrument technology and software radio technology, we realized to test the wireless sensor network IC automatically. Compared with other test system, this system has the advantages of high precision, high degree of automation and strong portability etc. It's fast and convenient to change test function and has a lowest cost of long-term use. This system can be used in the laboratory test and small batch automated test system, and also can be applied to large quantities of chip test production line.
\end{abstract}

\section{Introduction}

The technique of IOT (the abbreviation of Internet of Things) is becoming more and more mature after several years of rapid development, especially with the widespread of smart phones. Currently, IOT applications has been extended to a number of areas, including smart home, security monitoring, intelligent interconnect automobile, environmental monitoring, smart grid, industrial 4.0 and other fields. IDC made a prediction on IDC FutureScape hold on December 7, 2014: Today, more than 50\% of the behavior of IOT application focus on manufacturing, transportation, smart cities, consumer applications, but in the next five years, all the industry will launch initiatives on IOT. According to IDC predicts that by the year of 2020 more than 30 billion devices will access to the Internet of Things, the global networking market size will increase from $\$ 265.6$ billion in 2014 to $\$ 3.04$ trillion in 2020 [1]. With the rapid growth of the market size of IOT, related IC demand showing explosive growth.

Rapid growth in market demand accelerates technique upgrading. Popular chips in the market will be replaced by smaller chips with lower prices and lower power consumption in a few months. Types and quantities of IOT application chips showing explosive growth. Time to market of new designed chip is getting shorter. Traditional methods and procedures of IC design, manufacture and test can hardly adapt market demand. In particularly, chip test session play a more and more important part of IC industry.

Different chip testing methods are adopted in different segments of chip development.

R\&D staffs use general purpose bench test instruments, for example oscilloscope, signal source, spectrum analyzer and network analyzer, to set up a test environment to test their chips in preliminary stage of chip design. Bench test instruments can be easily used and have high test accuracy. Users can implement their test without additional complex programming. This method has high cost of test because general purpose test instruments are expensive.

Customized special equipments for particular test are adopted in chip manufacture stage. These special test equipments are highly customized according to test demands for chips. Function redundancy of general purpose test equipments is greatly reduced. Only necessary functions are left. So these customized equipments are low cost and compact. Customized equipments require longer 
development time. Only mature chips and communication protocols are worth to develop these special test equipments.

A compact virtual instrument test platform based on PXI architecture stand out to adapt new technical demand. This test platform has compact structure and low cost. Users can realize their test via programming customized software. Different test can be realized by modifying the software without changing hardware. This platform has high reusability of hardware. R \& D engineers only need to focus on test method design and test software programming when facing test requirement. This platform greatly reduces the time of test system development [2,3].

In this paper, we design a high precision automated test system for IOT chips based on the software and hardware platform mentioned above. We built an automated testing platform using programmable test equipments and designed a series of test boards to adapt the test process. Combined virtual instrument technology and software radio technology, we realized to test the wireless sensor network IC automatically.

\section{System design}

The block diagram of the high-precision automation networking chip test systems described in this paper is as follows:

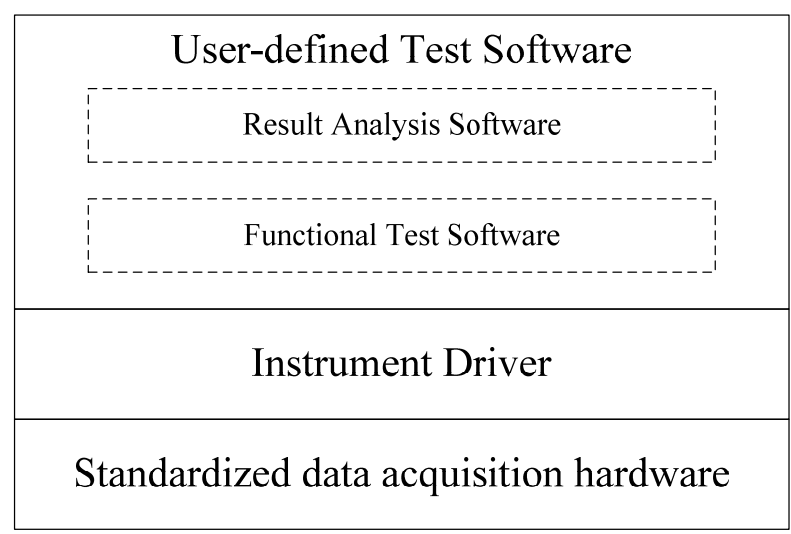

Fig. 1 Block diagram of the system

The system consists of standardized data acquisition hardware, instrument drivers, and user-defined test program three major components. The role of each part is as follows:

(1)Standardized data acquisition hardware

It realizes excitation signal generation, response signal acquisition, signal conditioning etc.

(2)Instrument driver

Instrument driver realizes to exchange data between the computer and the standardized data acquisition hardware. Instrument driver links the software and the programmable hardware. It is generally provided by the equipment manufacturers. Users can call the API directly when using appropriate functions.

(3) User-defined test program

Users write their own functional testing software and data analysis software based on their actual test requirements. User-defined test program control the standardized data acquisition hardware by calling instrument driver API to realize various test process. The test results can be analyzed automatically by user-defined data analysis software.

\section{Hardware Connection}

The RF signal of the test system is connected by a three-port RF circulator. The RF signal transmission path of three-port RF circulator is shown below: 


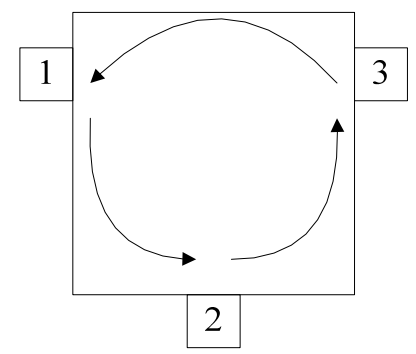

Fig. 2 Signal transmission path of three-port RF circulator

Signal input from port 1 can only output from port 2 rather than port 3 . Signal input from port 2 can only output from port 3 rather than port 1 . Signal input from port 3 can only output from port 1 rather than port $2[4,5,6]$. Three-port RF circulator make sure the signal transmitted according to a predetermined transmission path. No signal can transmit reverse the transmission path.

Hardware connections of the test system are as shown below:

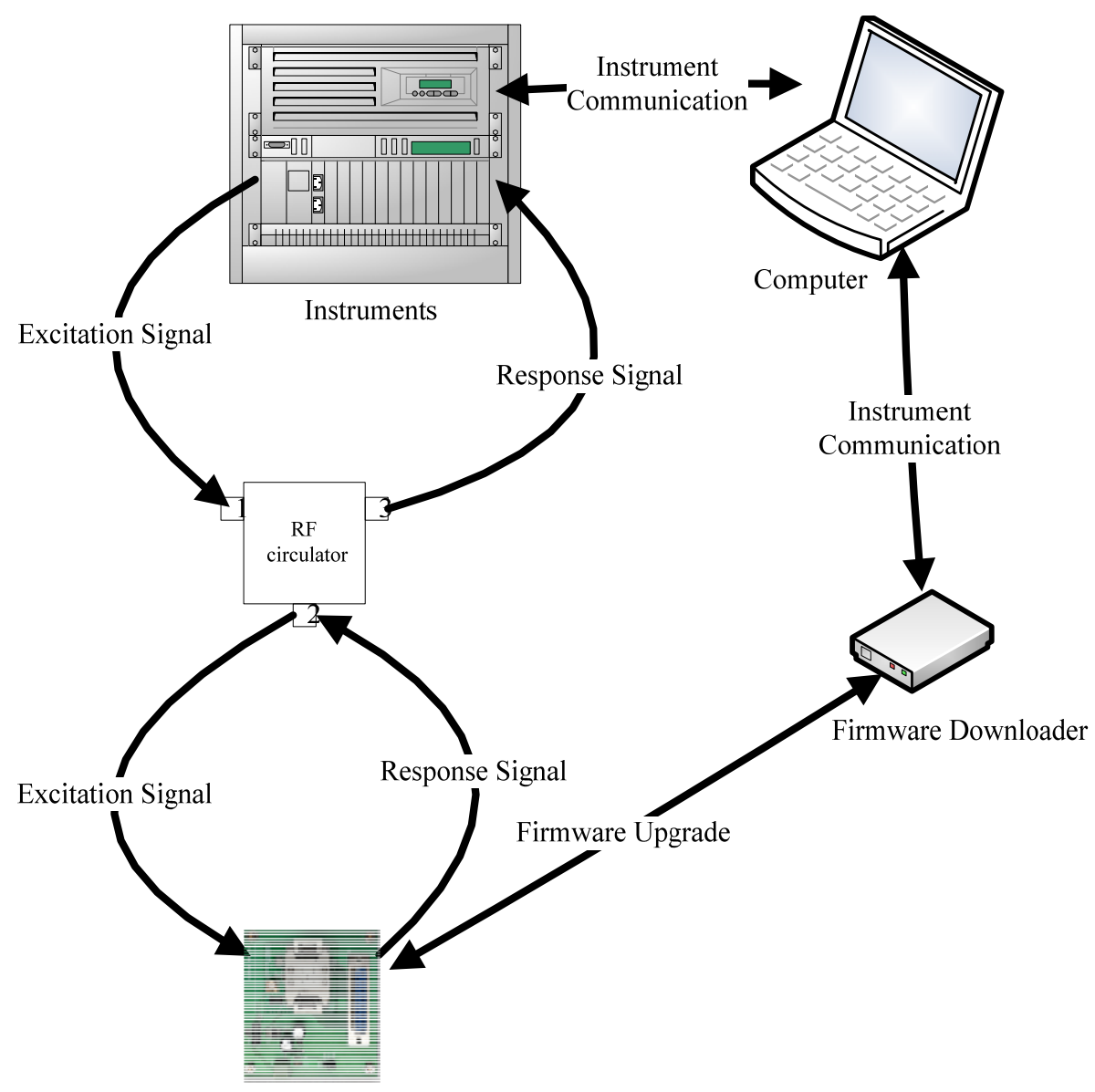

Unit Under Test

Fig. 3 Hardware connections of the test system

Computer is connected to the test instruments and firmware downloader. Firmware downloader is a device which is used to update the firmware of the unit under test. User-defined software running on the computer controls the firmware downloader to update the firmware of the unit under test and the test instruments to generate test excitation signal.

Signal output terminal of the test instrument is connected to the signal input port of the three-port RF circulator (port 1). Signal input from port 1 of the three-port RF circulator is transmitted to the unit under test via port 2. The response signal of the unit under test fed back to the three-port RF circulator via port 2. Signal input from port 2 of the three-port RF circulator is transmitted to the test instrument via port 3 .

Figure 4 shows the test system implements to test a unit under test. 


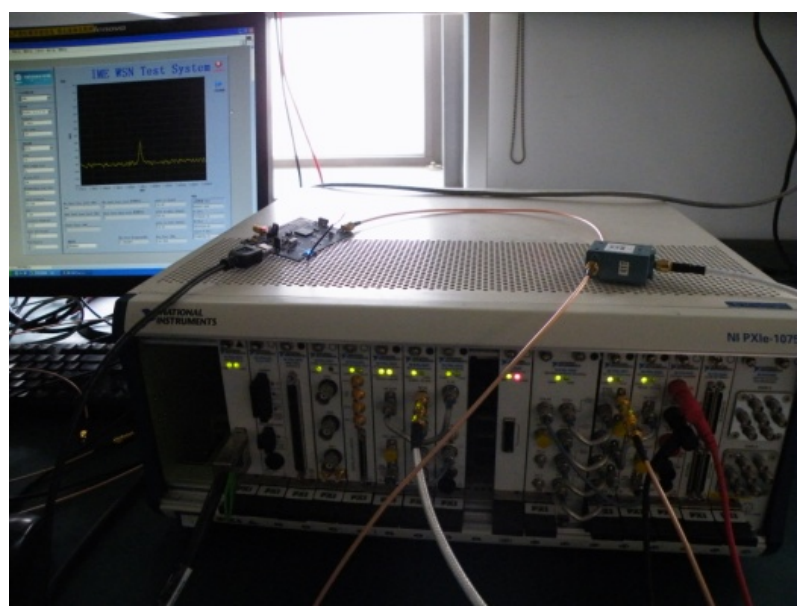

Figure 4 UUT Test

\section{Software Flow}

Software running on the test computer acquires the test data and analysis the data via user-defined algorithm.

Operation flow chart of the system is shown below:

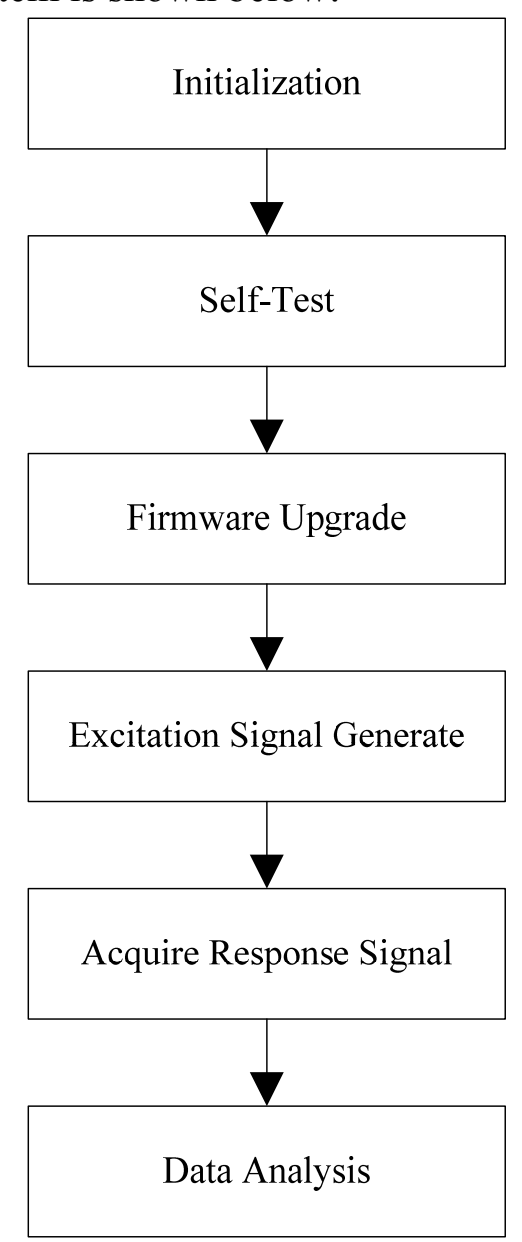

Fig. 5 Operation flow chart of the system

1. Some initialization work must be done after system starts. Some default configuration parameters, revise compensation parameters are loaded into the software.

2. The system will be self-test after initialization is complete. This will check whether the system test devices are connected and they work fine. 
3. User-defined software running on the test computer will update the firmware of the unit under test after the system self-test passes. Unit under test will be configured to be tested state by the firmware.

4. User-defined software running on the test computer controls the test instruments to generate the test excitation signal. Test excitation signal will be loaded to the unit under test through the three-port RF circulator.

5. Test excitation signal drives the unit under test to generate a response signal. Response signal transmits to the test instrument via three-port RF circulator. User-defined software running on the test computer collects and saves the response data.

6. User-defined software running on the test computer makes a comprehensive analysis to obtain test results according to the system configuration parameters, revise compensation parameters and actual test data.

\section{Test Board Design}

In order to meet the needs of the chip test, we design a test board to adapt the test process as shown below.

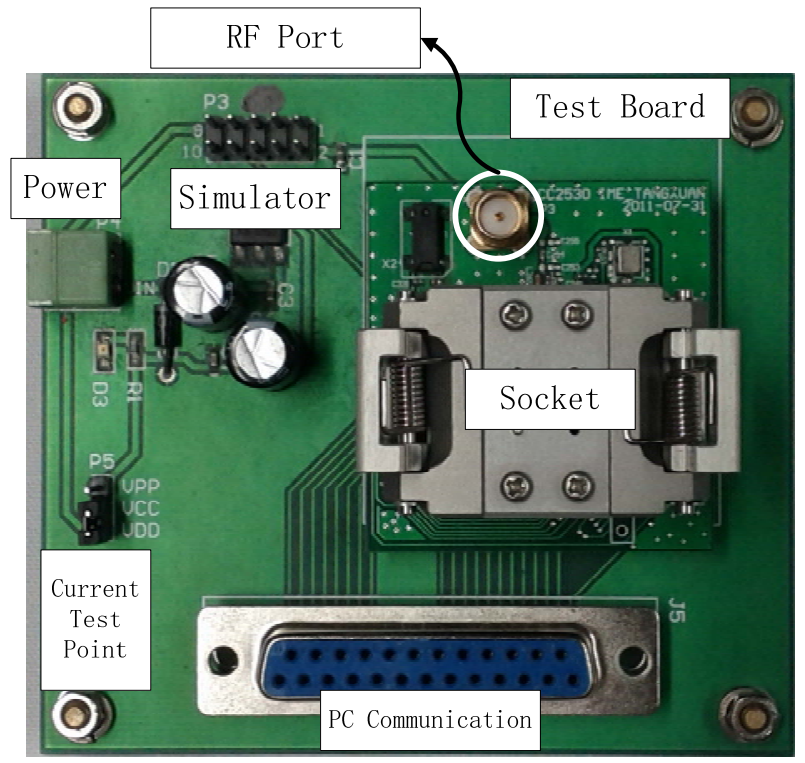

Fig. 6 Test Board

In this test board, we use a Socket as a chip test fixture. All the pins of the chip under test are drawn on the external pins of the Socket. We designed a matching circuit that allows the chip to work in a normal state. When the chip is mounted in the Socket, all the pins of the chip under test are connected to the external matching circuit through the Socket pins.[7,8]

The test board also provides a power supply, current test point, program download and simulation interface, computer communication port and other matching circuit.

\section{Summary}

This test system can be developed quickly and easily. Compared with other test systems, it has high test accuracy, high degree of automation and high portability. It has low cost when long-term use.

1. Use the three-port RF circulator to censure the transmission direction of the signal transmitted according to a predetermined transmission path. This ensure the test system work truly automatically without manually switch the signal as input or output.

2. The loss of three-port RF circulator to the instruments and the circuit boards and the loss of chip pin to the socket are considered. The RF parameters of chip pin to the SMA header of the circuit board are measuring by the network analyzer. All losses and other factors are taken into account. 
3. The use of chip socket ensures truly industrial pipelined test. The chip under test is placed in the socket, and can be replaced easily.

4. Firmware of the unit under test can be upgraded automatically. This improves the test speed and degree of automation.

The system can be used in laboratory tests and small quantities of automated tests. It also can be applied to high-volume chip test after some necessary modify. If with the mechanical hands and other automation devices the test system can be directly used in the production line to achieve online automatic testing. It has a strong industrial application value.

\section{Acknowledgments}

This work was supported by "High-density flip-chip package substrate design and verification" (NO. 2013ZX02502-001) which is the sub-project of "High-density packaging flip-chip substrate product development and industrialization" (NO. 2013ZX02502). This work was also supported by "Study on the electrical transport properties of differential TSV structure in the 3D (TSV) package" (NO. 61306135).

Research funding for the project comes from the sub-project of the Very Large Scale Integrated Circuits Manufacturing Equipment and Complete Technology which is NO.2 National Major Projects of China and National Natural Science Foundation of China.

\section{References}

[1] IDC: Announced in 2015 the world's top 10 forecast of Internet of Things, Electronic technology and software engineering, vol.1, 2015

[2] Barrera E, Ruiz M, Lopez S, et al. PXI-based architecture for real-time data acquisition and distributed dynamic data processing. IEEE Transactions on Nuclear Science, 2006, vol.53 (3), pp923-926.

[3] Fang Bao and Yan Feng, Virtual instrument testing system based on PCI / PXI / VXI bus. Industrial instrumentation and automation equipment,2000 (3), pp17-19.

[4] Information on https://en.wikipedia.org/wiki/Circulator

[5] Information on http://www.rf-circulator.com/index.html

[6] Information on http://www.everythingrf.com/browse/microwave-rf-circulators

[7] Mielke, J.A. and Pope, K.A., High-speed fixture interconnects for mixed-signal IC testing, Test Conference, 1990. Proceedings, International, pp891 - 895.

[8] Mehtani, K., Atzema, B., De Jonghe, M., Morren, R., Seuren, G. and Zwemstra, T., MixTest: A mixed-signal extension to a digital test system, Test Conference, 1993. Proceedings., International, pp945-953. 